Vibramycin (doxycycline hyclate capsule) $100 \mathrm{mg}$ orally twice daily for 7 days for the treatment of uncomplicated urogenital chlamydia was evaluated in a randomised, double-blind, double-dummy, activecontrolled, multicenter study. Males and nonpregnant females ages 19-45 with a confirmed diagnosis of urogenital chlamydia $<14$ days prior to enrolment or with a sexual partner with chlamydia were eligible. The study consisted of three visits: baseline, day 8 (end-oftreatment), and day 28 (test-of-cure [TOC]). The primary outcome was microbiological cure at TOC, defined as a negative result for Chlamydia trachomatis by the Gen-Probe (GP) Aptima Combo (AC) 2 assay (on urine in males and vaginal swab in females). The mITT population consisted of treated subjects with a GP AC2 positive for $C$ trachomatis at baseline. Non-inferiority of WC2031 was inferred if the lower limit of the $95 \%$ CI of the difference in cure rates was $>-10 \%$. Safety was studied through clinical evaluation and laboratory tests.

Results 495 subjects were randomised at 41 study sites. The mITT population with evaluable efficacy consisted of 323 (65\%) subjects (156 in the WC 2031 group and 167 in the Vibramycin group). Baseline characteristics did not differ by group: median age $23,61 \%$ female, 58\% African American vs 36\% Caucasian vs 7\% other race, and $21 \%$ Hispanic ethnicity. The microbiological cure rate (95\% CI) for the WC2031 group was $95.5 \%$ (92.3 to 98.8) vs $95.2 \%$ (92.0 to 98.4) for the Vibramycin group; the $95 \%$ CI for the difference in cure rates between treatments was ( $-4.3 \%$ to $4.9 \%)$. Types of adverse events were comparable between treatment groups. The WC 2031 group had less nausea and vomiting ( $13 \%$ vs $21 \%$ and $8 \%$ vs $12 \%$ ). Conclusions WC2031 was non-inferior to Vibramycin for treatment of uncomplicated urogenital chlamydia, was better tolerated, and demonstrated comparable safety. The once daily dosing of WC2031 may improve treatment adherence compared with twice daily doxycycline.

\section{3-S4.04 TIME TO CURE CHLAMYDIA: PROSPECTIVE STUDY ON DETECTION OF ANORECTAL AND CERVICOVAGINAL CHLAMYDIA AFTER DIRECTLY OBSERVED TREATMENT WITH AZITHROMYCIN}

doi:10.1136/sextrans-2011-050109.124

\begin{abstract}
${ }^{1,2} \mathrm{~N}$ Dukers-Muijrers, ${ }^{1} \mathrm{M}$ Werner, ${ }^{1} \mathrm{E}$ Brouwers, ${ }^{3} \mathrm{~A}$ Speksnijder, ${ }^{1} \mathrm{~L}$ Spauwen, ${ }^{3} \mathrm{~S}$ Bruisten, ${ }^{4} \mathrm{I}$ van den Broek, ${ }^{5} \mathrm{M}$ van der Sande, ${ }^{6} \mathrm{~S}$ Morré, ${ }^{1} \mathrm{C}$ Hoebe. ${ }^{1}$ Department of Infectious Diseases, South Limburg Public Health Service, Geleen, Netherlands; ${ }^{2}$ Department of Medical Microbiology, Maastricht University Medical Centre, School for Public Health and Primary Care, Geleen, Netherlands; ${ }^{3}$ Public Health Laboratory, Health Service Amsterdam, Amsterdam, Netherlands; ${ }^{4}$ Epidemiology and Surveillance, Centre for Infectious Diseases Control, National Institute for Public Health and Environment, Bilthoven, Netherlands; ${ }^{5}$ Epidemiology and Surveillance, Centre for Infectious Diseases Control, National Institute for Public Health and Environment and Julius Centre, UMCU, Bilhoven, Netherlands; ${ }^{6}$ VUmc, Department of Pathology, Laboratory of Immunogenetics, Amsterdam, Netherlands
\end{abstract}

Background When a person, diagnosed with Chlamydia trachomatis $(\mathrm{Ct})$, is being treated for Ct, STI Centres generally advise a short period of sexual abstinence; in most cases no test of cure is performed. These are considered best practices to cure $\mathrm{Ct}$ and prevent further spread, assuming high antibiotic efficacy. However, in about $5 \%$ of treated patients symptoms persist and among those retested $6-12$ months later, $10 \%$ is Ct positive (again). Moreover, the efficacy of Azithromycin treatment for anorectal Ct is debated. The current study aims to increase understanding $\mathrm{Ct}$ treatment efficacy and to estimate time to cure cervicovaginal and anorectal $\mathrm{Ct}$, using repeated measurements of laboratory, medical, and exposure parameters.

Methods Prospective study among 49 persons with cervicovaginal Ct (45 women) and/or anorectal Ct (four men, three women). After directly observed treatment with $1000 \mathrm{mg}$ Azithromycin, each participant provided during 2 months 18 time-sequential self-taken samples for testing for rRNA (TMA), Ct bacterial load (quantitative PCR; Ct plasmid DNA), and type Ct (serovar), and three timesequential questionnaires on potential exposure (behaviour) and symptoms. Steady partners were treated as well. Here, we report on the 905 rRNA tests in 49 patients.

Results Of all women with cervicovaginal Ct, the proportion rRNA positive was $100 \%$ at treatment, $54 \%$ at day $7,11 \%$ at day 12 and $17 \% 2$ months after treatment. For anorectal $\mathrm{Ct}$, these rates were $100 \%, 57 \%, 29 \%$, and $50 \%$. In total $45 \%$ of persons with cervicovaginal $\mathrm{Ct}$ and $57 \%$ with anorectal $\mathrm{Ct}$ had at least one positive rRNA sample in the period between 2 weeks and 2 months after treatment. Three cases of anorectal Ct (negative Lymfogranuloma venerum (LGV) PCR) remained rRNA positive during 2 months and tested $\mathrm{Ct}$ negative after administration of doxycycline (testing 2 months later).

Conclusions The proportion of Ct rRNA positivity during follow-up was much higher than expected in the light of assumed high effectiveness of Azithromycin treatment. Time to cure depends on the extent rRNA indicates clinically relevant or persistent $\mathrm{Ct}$ infection; this will be determined considering other markers (eg, $\mathrm{Ct}$ DNA) and symptoms as well (research ongoing). The persistent anorectal Ct cases that resolved after doxycycline treatment, contribute to the notion that this might be better treatment in anorectal Ct, after exclusion of LGV. Current study contributes to evidence based patient management and $\mathrm{Ct}$ transmission reduction.

\section{3-S4.05 TREATMENT OF STUDENTS INFECTED WITH CHLAMYDIA AND GONORRHOEA IN A SCHOOL-WIDE SCREENING PROGRAM}

doi:10.1136/sextrans-2011-050109.125

${ }^{1} \mathrm{~S} N$ Taylor, ${ }^{1} \mathrm{C}$ Brennan, ${ }^{2} \mathrm{~L}$ Longfellow, ${ }^{1} \mathrm{D}$ H Martin, ${ }^{1} \mathrm{M} J$ N Nuami. ${ }^{1}$ Louisiana State University Health Sciences Center, New Orleans, USA; ${ }^{2}$ Louisiana Office of Public Health, USA

Background Providing treatment to individuals screened for a health condition is a key component of any health screening program. School-based screenings for sexually transmitted diseases (STD) offer the possibility of efficiently and promptly treating participants because students can be easily located after they have been tested. We describe treatment rates and time to treatment for students with Chlamydia trachomatis (CT) or Neisseria gonorrhoeae (NG) infection in a school-wide screening program.

Methods Between 1995 and 2005, 20224 high school students were tested for CT and NG during annual screenings using urine specimens and commercial nucleic acid amplification tests. Test results were available approximately five working days after specimen collection. Students who tested positive were located at school during regular class hours for counselling and treatment by a school nurse, a clinic nurse, a public health nurse, or a physician. Treatment was with a single 1 gram oral dose of azithromycin for CT and $500 \mathrm{mg}$ oral ciprofloxacin for NG, administered under direct observation. Before its removal from the market, cefixime $400 \mathrm{mg}$ in single oral dose was used to treat NG. The names of infected students who could not be located in school were forwarded to a public health Disease Intervention Specialist (DIS) for follow-up. The DIS provided the program with an update on the follow-up status of each name referred.

Results During the 10-year period, 3422 infections (CT: 2746; NG: 304; CT and NG: 372) were identified. Treatment was documented for 2844 infections (83.1\%). There were no significant differences in rates of treatment $(p>0.37)$ by gender or by infection. The rates of treatment varied by school year from a low of 64.8\% in 1996-1997 
to a high of $94.9 \%$ in 1997-1998 ( $p<0.0001)$. For 2692 infections with documented dates of treatment, the median time from specimen collection to treatment was 21 days (75th percentile: 33 days).

Conclusions This high school STD screening and treatment program achieved high treatment rates for both CT and NG, although the treatment effort required strong commitments of various individuals from the screening program, the administrations in participating schools, and the local health services. Differences in treatment rates per school year reflected the dynamic interplay of these various individuals and organisations as well as the commonly high absentee, truancy, and dropout rates among students in the school district.

\section{3-S4.06 PREDICTORS OF CLINICAL TREATMENT FAILURE AMONG MEN WITH IDIOPATHIC NGU}

doi:10.1136/sextrans-2011-050109.126

${ }^{1} \mathrm{D}$ V Colombara, ${ }^{1} \mathrm{~L}$ E Manhart, ${ }^{1} \mathrm{C}$ M Wetmore, ${ }^{2} \mathrm{M}$. S Lowens, ${ }^{1} \mathrm{~N}$ A Kay, ${ }^{1} \mathrm{P}$ A Totten, ${ }^{2} \mathrm{M}$ R Golden. ${ }^{1}$ University of Washington Seattle, USA; ${ }^{2}$ Public Health-Seattle \& King County, University of Washington, Seattle, USA

Background Up to half of men with nongonoccocal urethritis (NGU) have no known aetiology, yet still receive syndromic treatment. Identifying characteristics associated with clinical treatment failure may aid in determining the aetiology of these cases.

Methods From 1 January 2007 to 31 December 2010, 553 men entered a randomised double-blind treatment trial for NGU at the Public Health Seattle \& King County STD clinic in Washington. Eligible men had visible urethral discharge or $\geq 5 \mathrm{PMNs} /$ high power field on a Gram stained slide of urethral exudates. Men were randomised to either $1 \mathrm{~g}$ single dose azithromycin or $100 \mathrm{mg}$ doxycycline twice daily for 7 days. Chlamydia trachomatis, Neisseria gonorrhoeae, and Trichomonas vaginalis were assessed by TMA (Gen-Probe, Inc., San Diego, CA); Mycoplasma genitalium was assessed by an in-house PCR assay. Ureaplasmas were detected by culture and speciated by a Ureaplasma urealyticum-biovar two specific PCR. Men negative for all pathogens were considered idiopathic and invited to return $2-5$ weeks after enrolment. Clinical treatment failure was defined as visible urethral discharge or $\geq 5$ PMNs. We evaluated baseline demographic and clinical characteristics, self-reported sexual history at enrolment, sexual practices between visits and depression as potential correlates of clinical treatment failure using log binomial regression.

Results Of the 430 (81\%) men with NGU who returned for followup, 202 (47\%) were considered idiopathic at baseline. Enrollees were $68 \%$ white and $27 \%$ black. Age ranged from 19 to 62 . Fifty-one men (25\%) with idiopathic NGU experienced clinical failure. In multivariate analyses, purulent discharge at enrolment more than doubled the risk of failure ( $\mathrm{ARR}=2.5,95 \% \mathrm{CI}: 1.4 \%$ to $4.4 \%$ ) and black men were nearly twice as likely as non-blacks to have treatment failure $(A R R=1.8,1.1$ to 2.8). Age, socioeconomic status, number of partners in last 2 months, sexual orientation, sexual behaviour (anal/ vaginal sex, unprotected sex between visits), depression, and other baseline clinical characteristics were not associated with treatment failure see Abstract O3-S4.06 table 1.

Conclusions Treatment failure was common among men with idiopathic NGU and associated with black race and purulent discharge at enrolment. The association with purulent discharge suggests an etiologic agent that evokes a robust immune response. Insofar as race defines sexual networks, an etiologic agent present in the network may explain the observed differential risk of persistent NGU.
Abstract 03-S4.06 Table 1 Measures of risk for clinical failure among men treated for idiopathic NGU

\begin{tabular}{|c|c|c|c|c|}
\hline \multirow{2}{*}{ Characteristics } & \multicolumn{2}{|l|}{ Univariate analysis } & \multicolumn{2}{|c|}{ Multivariate analysis } \\
\hline & RR (95\% Cl) & $\overline{p \text { Value }}$ & RR $(95 \%$ CI) & p Value \\
\hline \multicolumn{5}{|l|}{ Demographics } \\
\hline Age & 0.99 (0.97 to 1.02$)$ & 0.591 & & \\
\hline Black race & $1.66(1.02$ to 2.70$)$ & 0.042 & $\begin{array}{l}1.76(1.11 \text { to } \\
2.80)\end{array}$ & 0.016 \\
\hline Low socioeconomic status* & $1.24(0.76$ to 2.02$)$ & 0.394 & & \\
\hline \multicolumn{5}{|l|}{ Baseline signs and symptoms } \\
\hline PMNs $\geq 10$ & $1.40(0.85$ to 2.31$)$ & 0.190 & & \\
\hline Visible discharge & $1.66(0.65$ to 4.21$)$ & 0.286 & & \\
\hline Purulent discharge on exam & 1.75 (0.71 to 4.28$)$ & 0.223 & $\begin{array}{l}2.51(1.44 \text { to } \\
4.35)\end{array}$ & 0.001 \\
\hline Discharge amount on exam & & 0.789 & & \\
\hline Small & Referent & - & & \\
\hline Moderate & $1.18(0.70$ to 1.99$)$ & 0.536 & & \\
\hline Large & $0.87(0.25$ to 3.08$)$ & 0.834 & & \\
\hline Depression (CES-D score $\geq 10$ ) & $1.02(0.56$ to 1.87$)$ & 0.936 & & \\
\hline \multicolumn{5}{|l|}{ Sexual behaviours } \\
\hline Sex with a man in last 12 months & $0.94(0.56$ to 1.59$)$ & 0.819 & & \\
\hline $\begin{array}{l}\text { Any anal insertive partners } \\
\text { in last } 2 \text { months }\end{array}$ & 0.88 (0.51 to 1.53$)$ & 0.654 & & \\
\hline $\begin{array}{l}\text { Any vaginal sex partners } \\
\text { in last } 2 \text { months }\end{array}$ & $0.79(0.48$ to 1.29$)$ & 0.343 & & \\
\hline $\begin{array}{l}\text { Number sex partners in } \\
\text { last } 2 \text { months }\end{array}$ & $1.02(0.96$ to 1.09$)$ & 0.535 & & \\
\hline $\begin{array}{l}\text { Any unprotected sex } \\
\text { between visits }\end{array}$ & $1.06(0.56$ to 1.98$)$ & 0.864 & & \\
\hline
\end{tabular}

${ }^{*}$ As measured by ability to pay full price for the clinic visit vs payment on a sliding scale.

\section{Clinical sciences oral session 5-treatment: Syphilis, Herpes, \& Bacterial Vaginosis 03-S5.01 IMPACT OF AIC316, A NOVEL ANTIVIRAL HELICASE- PRIMASE INHIBITOR, ON GENITAL HSV SHEDDING: RANDOMISED, DOUBLE-BLIND, PLACEBO-CONTROLLED TRIAL}

doi:10.1136/sextrans-2011-050109.127

${ }^{1} \mathrm{~A}$ Wald, ${ }^{2} \mathrm{~S}$ Stoelben, ${ }^{3} \mathrm{~S}$ Tyring, ${ }^{4} \mathrm{~T}$ Warren, ${ }^{5} \mathrm{C}$ Johnston, ${ }^{1} \mathrm{M} \mathrm{L}$ Huang, ${ }^{2} \mathrm{~B}$ Timmler, ${ }^{2} \mathrm{H}$ Ruebsamen-Schaeff, ${ }^{6} \mathrm{~L}$ Corey, ${ }^{2} \mathrm{~A}$ Birkmann. ${ }^{1}$ University of Washington, Seattle, USA; ${ }^{2}$ AiCuris GmbH \& Co. KG Wuppertal, Germany; ${ }^{3}$ University of Texas Health Science Center Houston, USA; ${ }^{4}$ Westover Heights Clinic, Portland, USA; ${ }^{5}$ University of Washington, Seattle, USA; ${ }^{6}$ Fred Hutchinston Cancer Research Center, University of Washington, Seattle, USA

Background Current treatments for HSV infection are imperfect, do not completely abrogate viral shedding or transmission, and do not interrupt HSV-2-HIV interactions. AIC316 is a helicase-primase inhibitor, a new class of anti-HSV compounds that has a distinct mode of action from currently available nucleoside analogues.

Methods We investigated the safety and efficacy of AIC316 in patients with genital HSV-2 infection in a Phase 2, randomised, multicenter, parallel, double-blind, placebo-controlled trial of 4 different doses of AIC316 administered orally for 4 weeks. Participants were randomised 1:1:1:1:1 to one of the following dose groups: $5 \mathrm{mg}$ given once daily (qd), $25 \mathrm{mg}$ qd, $75 \mathrm{mg} \mathrm{qd}, 400 \mathrm{mg}$ given once-a-week, or matching placebo. Participants in the once daily dose groups received a loading dose. During the trial period participants obtained a swab of genital secretions daily and maintained a diary of genital lesions. The primary endpoint was the rate of genital HSV shedding measured by HSV DNA PCR in the AIC316 groups vs placebo. 